\title{
Amélioration des méthodes de dosage de traces d'hexane résiduel dans les huiles végétales raffinées
}

Improving methods for determining the quantity of trace elements of residual hexane in refined vegetable oils

Oléagineux, Corps Gras, Lipides. Volume 8, Numéro 2, 167-73, Mars - Avril 2001, Fondamental

Auteur(s) : Florence LACOSTE, Evelyne BRENNE, Jean-Louis COUSTILLE, Iterg, rue Monge, 33600 Pessac.

Résumé : Afin d'améliorer la sensibilité et la répétabilité du dosage des traces d'hexane résiduel dans les corps gras pour des teneurs de l'ordre $\mathrm{du} \mathrm{mg} / \mathrm{kg}$, une optimisation de la méthode décrite dans la norme Afnor NF T 60-257 a été entreprise en chromatographie en phase gazeuse. L'utilisation d'une colonne de type wide-bore à film épais, en couplage avec un appareil automatique d'espace de tête statique ou même en pratiquant l'analyse avec injection manuelle, permet de quantifier $0,25 \mathrm{mg} / \mathrm{kg}$ d'hexane technique. La linéarité de la réponse est très bonne dans le domaine de teneurs considéré, de 1 à $10 \mathrm{mg} / \mathrm{kg}$. Les taux de récupération sont satisfaisants puisqu'ils sont compris entre 93 et $107 \%$ pour les échantillons de concentration en hexane technique comprise entre 0,25 et $2 \mathrm{mg} / \mathrm{kg}$. Les résultats témoignent également de la bonne répétabilité de cette méthode ; en effet, pour une concentration de $1 \mathrm{mg} / \mathrm{kg}$, le coefficient de variation est d'environ $3 \%$.

Mots-clés : huiles végétales raffinées, résidus, solvant d'extraction, hexane technique, chromatographie en phase gazeuse, espace de tête statique.

Summary : In order to increase the sensitivity and repeatability of the determination of residual hexane content in fats and oils for levels of about $1 \mathrm{mg} / \mathrm{kg}$, an optimization of the procedure given in the Afnor standard NF T 60-257 was realized. Using a wide-bore gas chromatography column, coupled with an automatic headspace apparatus or even operating in manual injection, allows to quantify $0.25 \mathrm{mg} / \mathrm{kg}$ of technical hexane. Linearity of the response is very good in the considered scale, 1 to $10 \mathrm{mg} / \mathrm{kg}$. Recovery rates are satisfying, between 93 and 107\% for samples with levels between 0.25 and $2 \mathrm{mg} / \mathrm{kg}$ of technical hexane. The improved method has also a good repeatability ; relative standard deviation is about $3 \%$ for a $1 \mathrm{mg} / \mathrm{kg}$ concentration.

Keywords : refined vegetable oils, traces, extraction solvent, technical hexane, gas chromatography, static headspace. 


\section{ARTICLE}

L'hexane technique est d'un usage courant en extraction industrielle des oléagineux. Il permet de récupérer l'huile suite à l'aplatissage ou au broyage des graines (soja) ou celle restant dans le tourteau après l'étape de pression (colza, tournesol, etc.). En principe, le raffinage et, en particulier, la désodorisation sont censés éliminer la totalité de ce mélange d'hydrocarbures constitué principalement de $n$-hexane et en moindre proportion de 2-méthyl-pentane, 3-méthyl-pentane, méthyl-cyclopentane et cyclohexane.

La législation française concernant les résidus d'hexane dans les corps gras (graisses, huiles et beurre de cacao) n'a cessé d'évoluer depuis le début des années 70. En effet, la teneur maximale en hexane technique fixée à $50 \mathrm{mg} / \mathrm{kg}$ par l'arrêté de 1973 [1] a été abaissée à $5 \mathrm{mg} / \mathrm{kg}$ en 1990 [2] suite à un rapprochement des législations des États membres de la CEE. Cette valeur limite est actuellement de $1 \mathrm{mg} / \mathrm{kg}$ depuis 1998 [3].

Aujourd'hui, les méthodes d'analyse normalisées pour ce dosage, sur le plan international ou au niveau national, ont des performances restant en deçà de cette exigence. En effet, la norme NF EN ISO 9832 [4] ne convient que pour des teneurs supérieures ou égales à $10 \mathrm{mg} / \mathrm{kg}$, alors que la norme NF T 60-257 [5] permet de doser les faibles quantités d'hexane à partir de $2 \mathrm{mg} / \mathrm{kg}$.

L'objectif de notre étude a été d'améliorer la sensibilité et la précision de ces méthodes de dosage de façon à couvrir un domaine de concentrations de 0,5 à $10 \mathrm{mg} / \mathrm{kg}$. À cet effet, deux appareils automatiques de génération d'espace de tête ont été testés, de même que l'utilisation d'une colonne capillaire de type wide-bore imprégnée d'un film épais autorisant l'injection de volumes gazeux plus importants. De plus, la possibilité de réaliser l'analyse avec une injection manuelle a été vérifiée, ceci afin de permettre aux laboratoires réalisant seulement des contrôles ponctuels de s'affranchir de l'investissement d'un appareil automatique.

\section{Travail expérimental}

\section{Matériel}

Nous avons pu disposer et mettre en œuvre deux appareils pour génération d'espace de tête selon le mode statique travaillant de façon automatique en gardant comme référence la technique manuelle avec flacon et seringue à gaz.

\section{Descriptif de l'appareil HS 500 (de CTC Analytics AG)}

Disposant de ce matériel au laboratoire, nous l'avons couplé avec un chromatographe en phase gazeuse afin de réaliser une série essais. L'appareil est composé :

- d'un plateau permettant de recevoir 50 flacons de $10 \mathrm{ml}$ dans lesquels sont placés les échantillons ;

- d'un four réglé thermiquement (de 30 à $120^{\circ} \mathrm{C}$ ), muni d'un système d'agitation et pouvant admettre deux flacons ;

- d'un système mobile de prélèvement automatique de l'échantillon gazeux à l'aide d'une seringue chauffée, de capacité maximale de $2,5 \mathrm{ml}$. 


\section{Descriptif de l'appareil HS 40 XL (de Perkin Elmer)}

Les travaux publiés par Ollivier en 1996 et 2000 [6, 7] font état, sur ce type d'appareil, d'une sensibilité accrue par rapport à la méthode décrite dans la norme NF T 60-257. Ayant pu disposer momentanément de ce matériel, nous avons souhaité en tester les possibilités. Il se compose :

- d'un carrousel permettant l'analyse de 40 échantillons (flacons de 10 ou 20 ml) ;

- d'un four régulé pouvant contenir quatre flacons ;

- d'un dispositif comportant une aiguille mobile permettant la pressurisation du flacon puis l'évacuation de la fraction gazeuse de l'échantillon vers le chromatographe ;

- d'une ligne de transfert thermostatée (volume mort réduit : 50 mul).

Le principe de fonctionnement en est le suivant :

Étape 1 : Génération de l'espace de tête par chauffage de l'échantillon.

Étape 2 : La pressurisation du flacon à l'aide du gaz vecteur intervient, via l'aiguille pénétrant alors à travers le septum. Durant les quelques minutes de la phase de pressurisation, l'aiguille reste en position « basse ».

Étape 3 : Ensuite, grâce à l'action d'un jeu de vannes situé en amont de l'aiguille, l'arrivée du gaz vecteur se trouve en position fermée et l'échantillon gazeux sous pression est injecté dans la colonne de chromatographie sous l'effet de la détente. Les vannes sont ensuite réouvertes de façon à introduire le gaz vecteur dans le flacon, ce qui a pour effet de stopper l'injection.

Les paramètres dont les valeurs peuvent être modifiées sont nombreux : durée et température de l'étape de désorption, températures de l'aiguille et de la ligne de transfert, durée d'injection (fixant le volume injecté), durée de pressurisation, durée de retrait de l'aiguille après injection, pression d'hélium (déterminée par le débit en sortie colonne).

\section{Modes opératoires}

\section{Adaptation à l'injection manuelle des conditions d'analyse utilisées avec le HS 40 XL}

Les conditions chromatographiques utilisées avec le HS 40 XL ont été conservées : volume gazeux injecté de 2,5 ml, colonne de type wide-bore, programme thermique, débit du gaz vecteur.

Les conditions de génération de l'espace de tête (température, durée, prise d'essai) ont été volontairement calquées sur celles des normes existantes [4, 5].

\section{Récapitulatif des caractéristiques des méthodes testées}

Le tableau 1 présente un récapitulatif des conditions opératoires concernant:

- la méthode décrite dans la norme NF T 60-257

- l'utilisation de l'appareil HS 500 (de CTC Analytics AG) 
- I'utilisation de l'appareil HS 40 XL (de Perkin Elmer) couplé à un chromatographe équipé d'une colonne de type wide-bore

- la méthode fondée sur l'injection manuelle et l'utilisation d'une colonne de type wide-bore.

\section{Résultats}

\section{Évaluation de l'appareil HS 500 (de CTC Analytics AG)}

Une comparaison entre l'injection manuelle selon la norme NF T 60-257 et l'utilisation du HS 500 a été réalisée avec des échantillons contenant des teneurs en hexane technique de 1 à $10 \mathrm{mg} / \mathrm{kg}$. Les résultats montrent que le système automatique fournit une réponse inférieure à celle obtenue en injectant manuellement les échantillons (figure 1). Rappelons que la seule différence réside dans la taille des flacons, mais que le rapport prise d'essai/volume du flacon a été maintenu identique.

La perte de signal en utilisant le système automatique, comparativement à l'injection manuelle, est estimée à $40 \%$. La seringue à gaz du HS 500 n'étant pas équipée de vanne, il se pourrait qu'une partie de l'échantillon gazeux s'échappe de la seringue lors de son déplacement, entre le prélèvement et l'injection. Cette hypothèse pourrait également justifier la différence du facteur d'étalonnage $\mathrm{F}$ mise en évidence entre les deux modes d'injection. Celui-ci a été déterminé, pour ces deux modes d'injection, en respectant le protocole d'étalonnage décrit dans la norme NF T 60-257 (préparation des échantillons d'étalonnage, calcul du facteur d'étalonnage). En utilisant l'appareil HS 500 , le facteur F augmente comparativement à l'injection manuelle (tableau 2) ; I'hexane plus volatil est partiellement éliminé par rapport à l'heptane.

Une évaluation des taux de récupération a été réalisée avec des échantillons préparés par dilutions successives d'une huile raffinée complémentée à $100 \mathrm{mg} / \mathrm{kg}$. Les concentrations étudiées sont inférieures ou égales à $1 \mathrm{mg} / \mathrm{kg}$. Dans ce domaine de concentration, l'utilisation de l'appareil HS 500 conduit à un surdosage puisque les taux de récupération sont systématiquement supérieurs à $110 \%$, ce qui n'est pas le cas avec la méthode décrite dans la norme NF T 60-207 (tableau 3).

En utilisant la méthode décrite dans la norme NF T 60-257 (injection manuelle), on note que, pour l'échantillon contaminé à $0,25 \mathrm{mg} / \mathrm{kg}$, seuls trois des isomères de l'hexane technique sont détectés : les méthyl-2- et méthyl-3-pentane et le $\mathrm{n}$-hexane. Il faut une concentration d'au moins $1 \mathrm{mg} / \mathrm{kg}$ pour que les cinq isomères soient détectés. Ceci explique que la limite de quantification soit fixée à 2 $\mathrm{mg} / \mathrm{kg}$ dans cette norme.

\section{Évaluation de l'appareil HS 40 XL (de Perkin Elmer)}

Comparativement aux conditions de génération d'headspace préconisées par la norme, nous avons réduit la durée de désorption. En effet, entre 15 et 60 minutes, aucune augmentation du signal n'est observée (figure 2). Sachant par expérience qu'il est nécessaire d'attendre 15 minutes pour atteindre l'équilibre thermique, nous avons fixé la durée de désorption à 30 minutes.

En examinant la droite d'étalonnage (figure 3), il apparaît que la réponse est environ deux fois supérieure à celle obtenue lors des essais réalisés selon la norme NF T 60-257 (injection manuelle), ce qui n'est pas surprenant puisque la quantité injectée est 2,4 fois supérieure. 
Le facteur d'étalonnage a été déterminé en respectant le protocole d'étalonnage décrit dans la norme NF T 60-257 (préparation des échantillons d'étalonnage, calcul du facteur d'étalonnage) : il est de 0,48 .

Afin d'évaluer la sensibilité de l'appareil, différents échantillons ont été préparés par dilutions successives, à partir d'une huile raffinée complémentée à $100 \mathrm{mg} / \mathrm{kg}$, jusqu'à obtenir des échantillons d'huile de teneurs faibles comprises entre 0,10 et $0,95 \mathrm{mg} / \mathrm{kg}$ d'hexane technique.

La figure 4, présentant les profils chromatographiques obtenus pour l'huile de dilution et un échantillon complémenté à hauteur de $0,10 \mathrm{mg} / \mathrm{kg}$, montre que la limite de détection peut être fixée à $0,10 \mathrm{mg} / \mathrm{kg}$. En effet, la somme des surfaces correspondant aux isomères de l'hexane technique est alors environ 2,6 fois plus élevée que celle obtenue pour l'huile de dilution.

À partir des résultats obtenus pour ces échantillons volontairement chargés en hexane, le taux de récupération a été calculé (tableau 4). On remarque que l'huile de dilution contient environ 60 mug/kg d'hexane technique. En ce qui concerne les taux de récupération, les valeurs sont comprises entre 88 et $103 \%$, ce qui est tout à fait satisfaisant compte tenu des faibles teneurs étudiées. Le coefficient de variation de la concentration en hexane technique est compris entre 1,4 et $5 \%$ pour des teneurs entre 0,25 et $1 \mathrm{mg} / \mathrm{kg}$.

Ceci montre qu'il est tout à fait possible de doser correctement des teneurs inférieures à $1 \mathrm{mg} / \mathrm{kg}$ en utilisant un générateur automatique d'espace de tête statique couplé à une colonne wide-bore. Rappelons que, dans la norme NF T 60-257, le coefficient de variation de répétabilité est compris entre 3 et $5 \%$ pour une teneur de $2 \mathrm{mg} / \mathrm{kg}$.

\section{Application de la méthode sur colonne wide-bore avec injection manuelle}

Les étapes suivantes ont été réalisées : vérification de la linéarité de la réponse, évaluation du taux de récupération, estimation de la répétabilité, rédaction sous forme pré-normative du texte de la méthode d'analyse utilisée (annexe).

La linéarité de la réponse, vérifiée en réalisant une gamme d'étalonnage selon la méthode proposée (annexe), est excellente dans le domaine considéré, 1 à $10 \mathrm{mg} / \mathrm{kg}$ (figure 5). Le facteur d'étalonnage est identique à celui obtenu lors des essais réalisés avec l'appareil automatique HS $40 \mathrm{XL}$, soit de 0,48 .

Afin d'évaluer les taux de récupération, des échantillons d'huile de tournesol complémentés en hexane technique ont été préparés par dilutions successives : solution à $1000 \mathrm{mg} / \mathrm{kg}$ dans l'huile, puis à $100 \mathrm{mg} / \mathrm{kg}$, puis à $10 \mathrm{mg} / \mathrm{kg}$, puis enfin dilution de la solution à $10 \mathrm{mg} / \mathrm{kg}$ entre $0,1 \mathrm{et} 1 \mathrm{mg} / \mathrm{kg}$. Les résultats présentés dans le tableau 5 montrent que les taux de récupération sont satisfaisants puisqu'ils sont compris entre 93 et $107 \%$ pour les échantillons de concentration en hexane technique comprise entre 0,25 et $2 \mathrm{mg} / \mathrm{kg}$. Pour l'échantillon à $0,1 \mathrm{mg} / \mathrm{kg}$, ce taux est un peu plus fort (112\%).

La répétabilité de la méthode a été testée pour des taux d'incorporation d'hexane technique de 0,25 et $1 \mathrm{mg} / \mathrm{kg}$. Les résultats témoignent que l'injection manuelle, lorsqu'elle est bien maîtrisée, peut conduire à des résultats aussi répétables qu'avec un appareil automatisé (tableau 6). 
En effet, pour une concentration de $1 \mathrm{mg} / \mathrm{kg}$, le coefficient de variation est identique à celui obtenu avec l'appareil HS $40 \mathrm{XL}$, soit d'environ $3 \%$.

\section{Annexe \\ Méthode d'essai sous forme pré-normative - Corps gras d'origines animale et végétale - Dosage de faibles quantités résiduelles d'hexane technique}

\section{Domaine d'application}

Le présent document décrit une méthode de dosage de faibles quantités résiduelles d'hexane technique dans les huiles végétales raffinées.

Elle permet le dosage de teneurs en hexane technique comprises entre $0,2 \mathrm{mg}$ et $10 \mathrm{mg}$ par kilogramme de corps gras.

\section{Références normatives}

NF EN ISO 661: 1995, Corps gras d'origines animale et végétale. Préparation de l'échantillon pour essai.

\section{Principe}

Désorption des hydrocarbures volatils par chauffage à $80{ }^{\circ} \mathrm{C}$ dans un récipient fermé, après ajout d'un étalon interne. Dosage des hydrocarbures volatils par technique d'espace de tête statique et chromatographie en phase gazeuse sur colonne capillaire wide-bore.

\section{Réactifs}

Sauf indication contraire tous les réactifs doivent être de qualité connue.

4.1 Étalon interne, $n$-heptane exempt d'hexane (pureté > $99 \%$ ).

4.2 Gaz vecteur : hydrogène ou hélium ou azote (renfermant moins de $10 \mathrm{mg} / \mathrm{kg}$ d'oxygène).

4.3 Gaz auxiliaires : hydrogène (pur à $99,9 \%$ ) et air exempts d'impuretés organiques.

4.4 Hexane technique, d'une composition analogue à celle de l'hexane utilisé dans les méthodes de traitement industriel.

4.5 Huile végétale, raffinée et désodorisée, ayant une teneur en hexane technique inférieure à 0,1 $\mathrm{mg} / \mathrm{kg}$.

4.6 n-octane, exempt d'hexane et d'heptane (pureté > $99 \%$ ).

\section{Appareillage}

\subsection{Chromatographe en phase gazeuse à colonne capillaire}

5.1.1 Colonne capillaire, de $30 \mathrm{~m}$ de longueur, de phase stationnaire type polysiloxane apolaire ou faiblement polaire (DB 1, CPSil 5 CB ou similaire), à film d'épaisseur égale à 5 mum et diamètre 
intérieur de 0,53 $\mathrm{mm}$.

\subsubsection{Détecteur à ionisation de flamme.}

\subsubsection{Système d'acquisition de données ou intégrateur.}

5.1.4 Appareil de génération d'espace de tête statique multi-échantillon et d'injection automatique (facultatif).

\subsection{Matériel de laboratoire}

5.2.1 Capsules sertissables et déchirables en aluminium.

5.2.2 Enceinte thermostatée à $80^{\circ} \mathrm{C}$.

5.2.3 Fioles jaugées, classe $A$, de $100 \mathrm{ml}$.

5.2.4 Flacons en verre blanc (vial) de $25 \mathrm{ml}$, type « pénicilline » à sertir.

\subsubsection{Pince à sertir.}

5.2.6 Pipettes jaugées, classe A, deux traits, de $1 \mathrm{ml}$.

5.2.7 Seringues de 10 mul, utilisées individuellement pour préparer la gamme d'étalonnage et introduire l'étalon interne.

5.2.8 Seringue à gaz de $5 \mathrm{ml}$, équipée d'une vanne, utilisée uniquement pour l'analyse des solvants résiduels.

5.2.9 Septa en butyl avec disque téflon, inertes aux corps gras et aux solvants.

\subsubsection{Vortex}

\section{6. Échantillonnage}

Il est important que le laboratoire reçoive un échantillon réellement représentatif, non endommagé ou modifié lors du transport et de l'entreposage. L'échantillonnage ne fait pas partie de la méthode spécifiée dans le présent document. Une méthode d'échantillonnage recommandée est donnée dans la norme NF EN ISO 5555.

Il est indispensable que l'échantillon soit protégé d'un gain ou d'une perte de solvant.

\section{Préparation de l'échantillon à analyser}

Préparer l'échantillon pour essai conformément à la norme NF EN ISO 661.

\section{Mode opératoire}

\subsection{Conditions chromatographiques}

Les conditions d'analyses chromatographiques sont choisies en tenant compte des caractéristiques 
de la colonne et de la nature du gaz vecteur.

Par exemple :

- la température du four est maintenue à $50{ }^{\circ} \mathrm{C}$ pendant 8 minutes puis programmée, à raison de $10^{\circ} \mathrm{C} / \mathrm{min}$, jusqu'à $120^{\circ} \mathrm{C}$, la colonne est ensuite maintenue à $120^{\circ} \mathrm{C}$ pendant 5 min puis refroidie à $50{ }^{\circ} \mathrm{C}$;

- la température du détecteur est maintenue à $150^{\circ} \mathrm{C}$ et celle de l'injecteur à $200^{\circ} \mathrm{C}$.

La pression du gaz vecteur est ajustée de façon à chromatographier le $n$-hexane en 3 min et à maintenir le débit du gaz dans la colonne à environ $13 \mathrm{ml} / \mathrm{min}$.

La fuite de l'injecteur diviseur est d'environ $12 \mathrm{ml} / \mathrm{min}$.

Le détecteur à ionisation de flamme est réglé sur sa sensibilité maximale.

\subsection{Préparation des solutions d'étalonnage}

8.2.1 Solution d'hexane technique à $6,70 \mathrm{mg} / \mathrm{ml}$. Prélever exactement $1 \mathrm{ml}$ d'hexane technique (4.4) dans une fiole jaugée de $100 \mathrm{ml}$ (5.2.3) et compléter au volume avec l'octane (4.6).

8.2.2 Solution d'heptane à $6,84 \mathrm{mg} / \mathrm{ml}$. Prélever exactement $1 \mathrm{ml}$ d'heptane (4.1) dans une fiole jaugée de $100 \mathrm{ml}$ (5.2.3) et compléter au volume avec l'octane (4.6).

\subsection{Préparation de la gamme d'étalonnage}

Utiliser comme support et comme témoin à blanc une huile raffinée, par exemple une huile de tournesol (4.5).

Peser exactement 5,00 g ( $\pm 0,02 \mathrm{~g}$ ) d'huile végétale (4.5) dans six flacons (5.2.4). À l'aide d'une seringue (5.2.7), introduire dans chacun des flacons les volumes de solution d'hexane technique (8.2.1) et de solution d'heptane (8.2.2) indiqués dans le tableau.

Fermer chacun des flacons avec un septum (5.2.9) et une capsule métallique (5.2.1) et homogénéiser chaque flacon au vortex (5.2.10) en prenant soin de ne pas mettre du corps gras sur le septum.

\subsection{Préparation de l'échantillon à analyser}

Peser exactement 5,00 g ( $\pm 0,02 \mathrm{~g})$ d'huile à analyser. Ajouter 5,0 mul de solution d'heptane (8.2.2). Fermer chacun des flacons avec un septum (5.2.9) et une capsule métallique (5.2.1). Homogénéiser chaque flacon au vortex (5.2.10) en prenant soin de ne pas mettre du corps gras sur le septum (5.2.9). Chaque essai sera effectué en double.

\subsection{Désorption des volatils organiques}

Placer, toutes les $20 \mathrm{~min}$, un flacon dans l'enceinte thermostatée à $80{ }^{\circ} \mathrm{C}$ et le maintenir pendant $60 \mathrm{~min}$. 


\subsection{Analyse chromatographique des étalons et des échantillons}

À l'aide d'une seringue (5.2.8) chauffée à $60^{\circ} \mathrm{C}$, prélever $2,5 \mathrm{ml}$ de phase gazeuse située au-dessus du corps gras et injecter immédiatement dans le chromatographe.

Note : dans le cas de l'utilisation d'un appareil de génération d'espace de tête statique, adapter les quantités d'échantillon et volumes d'injection selon les caractéristiques de l'appareil et l'optimisation préalablement faite.

\subsection{Identification}

Dans les conditions chromatographiques décrites en (8.1), le chromatogramme de la solution étalon (figure 4) présente :

1 - cinq pics de temps de rétention voisins de 2,56, 2,84, 3,18, 3,92, 5,02 min correspondant respectivement aux isomères de l'hexane technique (4.4) ;

2 - un pic de temps de rétention voisin de 6,77 min correspondant à l'heptane (4.1).

\section{Détermination du facteur d'étalonnage}

Détermination du facteur d'étalonnage pour chacune des solutions d'étalonnage (tableau) enrichies en hexane technique, selon la formule suivante :

$$
F=\frac{C h \times \text { Aei }}{\left(A^{\prime} e c h-A b l\right) \times C e i}
$$

où :

Aéch : somme des aires des pics correspondant aux isomères de l'hexane technique (4.4) dans les solutions d'étalonnage.

Abl : somme des aires des pics correspondant aux isomères de l'hexane technique (4.4) présents dans l'huile végétale (4.5) utilisée pour la préparation de la gamme d'étalonnage (8.2.4).

Aei : aire du pic correspondant à l'heptane (4.1) dans les solutions d'étalonnage.

Ch : concentration en hexane technique, dans les solutions d'étalonnage (8.2.4), exprimée en $\mathrm{mg} / \mathrm{kg}$.

Cei : concentration en heptane, dans les solutions d'étalonnage (8.2.4), exprimée en $\mathrm{mg} / \mathrm{kg}$.

Exprimer les résultats avec deux décimales.

Calculer la valeur de la moyenne arithmétique $\mathrm{F}$ des cinq échantillons d'étalonnage qui doit être proche de 0,45. Le coefficient de variation obtenu doit être inférieur à $15 \%$.

\section{Expression des résultats}

La teneur en hexane technique résiduel dans l'échantillon à analyser, $W$, exprimée en $\mathrm{mg} / \mathrm{kg}$, est 
donnée par la formule suivante :

$$
W=\frac{A^{\prime} \dot{e ́ c h} \times F \times C e i}{A^{\prime} \text { ei }}
$$

où :

A'éch : somme des aires des pics correspondant aux isomères de l'hexane technique présents dans l'échantillon.

A'ei : aire du pic correspondant à l'heptane (8.4).

F : moyenne arithmétique du facteur d'étalonnage (9).

Cei : concentration en heptane, dans les solutions étalons (8.2.4), exprimée en $\mathrm{mg} / \mathrm{kg}$ c'est-à-dire $6,84 \mathrm{ppm}$.

\section{CONCLUSION}

L'objectif de ces travaux était l'amélioration de la sensibilité et de la répétabilité du dosage des traces d'hexane dans les corps gras pour des teneurs de l'ordre de la ppm. Pour cela, deux systèmes automatiques de génération d'espace de tête ont été testés au laboratoire.

La configuration de l'appareil HS 500 de CTC Analytics AG ne nous paraît pas optimale. En effet, le signal obtenu est nettement plus faible que lorsque l'injection est faite manuellement, cela dans les mêmes conditions d'analyse.

L'appareil HS 40 XL de Perkin Elmer a été volontairement associé à une colonne de type wide-bore pour permettre l'injection de volumes gazeux plus élevés. En augmentant le volume d'échantillon gazeux de 1 à 2,4 $\mathrm{ml}$ il devient possible de quantifier, avec exactitude et avec une précision suffisante ( $C V$ de $5 \%$ ), la teneur en hexane technique à partir de $0,25 \mathrm{mg} / \mathrm{kg}$. Ce matériel est d'un intérêt évident, gain de temps, bonne sensibilité, dans des unités qui doivent assurer des contrôles en série.

Ces essais ont également prouvé les performances des colonnes wide-bore imprégnées d'un film épais. Nous avons vérifié que cette configuration laissait aussi la possibilité de réaliser l'analyse avec injection manuelle. Dans ce cas, les résultats sont également satisfaisants puisque, pour une concentration de $1 \mathrm{mg} / \mathrm{kg}$, le coefficient de variation est d'environ $3 \%$.

Le texte de la méthode a été rédigé sous forme pré-normative. Au cours de l'année 2001, une analyse circulaire sera organisée par l'Iterg, en relation avec l'Afnor, afin d'obtenir des valeurs de fidélité pour cette nouvelle méthode qui sera ensuite proposée à la normalisation. 


\section{REFERENCES}

1. Arrêté du 12 février 1973. Liste des substances dont l'emploi est autorisé pour le raffinage et la transformation des corps gras alimentaires (JO du 15.02.1973).

2. Arrêté du 19 novembre 1990. Relatif aux solvants d'extraction utilisés dans la fabrication des denrées alimentaires ou de leurs ingrédients ( $J O$ du 06.12.1990).

3. Arrêté du 22 juin 1998 modifiant celui du 19 novembre 1990 (JO du 21.07.1998).

4. Norme NF EN ISO 9832 (2000). Corps gras d'origines animale et végétale. Dosage de I'hexane technique résiduel.

5. Norme NF T 60-257 (1998). Corps gras d'origines animale et végétale. Dosage de faibles quantités d'hexane technique résiduel.

6. OLLIVIER D, BARRIDAS S, GUERERE M (1996). Mise au point du dosage de faibles teneurs d'hexane résiduel dans les huiles. Ann Fals Exp Chim, 89 : 227-33.

7. OLLIVIER D, SOUILLOL S, GUERERE M (2000). Détermination de faibles teneurs d'hexane résiduel dans les huiles végétales. Ann Fals Exp Chim 93 : 111-4.

Illustrations

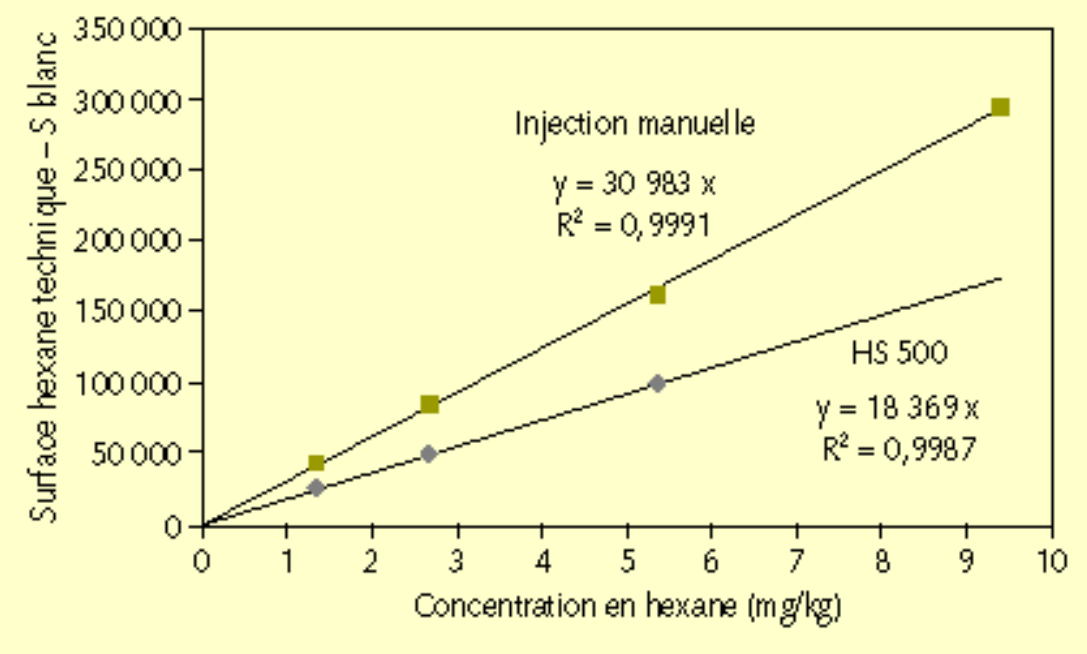

Figure 1. Comparaison des réponses chromatographiques obtenues en injection manuelle et avec le HS 500. 


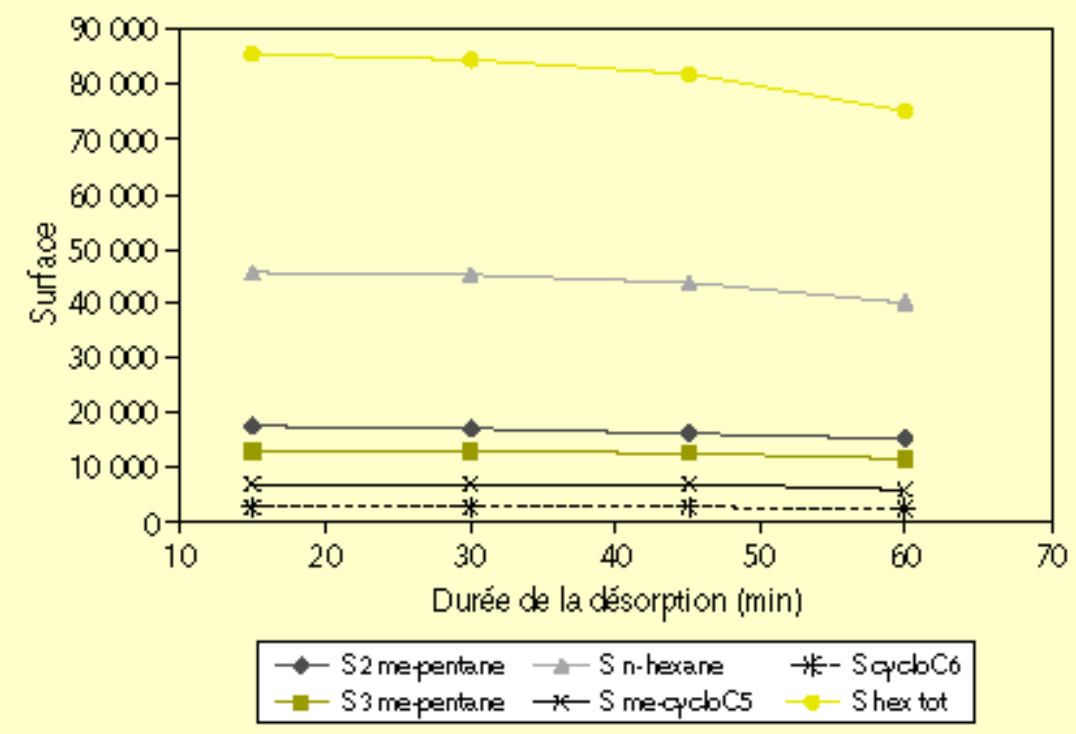

Figure 2. Appareil HS 40 XL - Influence de la durée de désorption sur la réponse chromatographique.

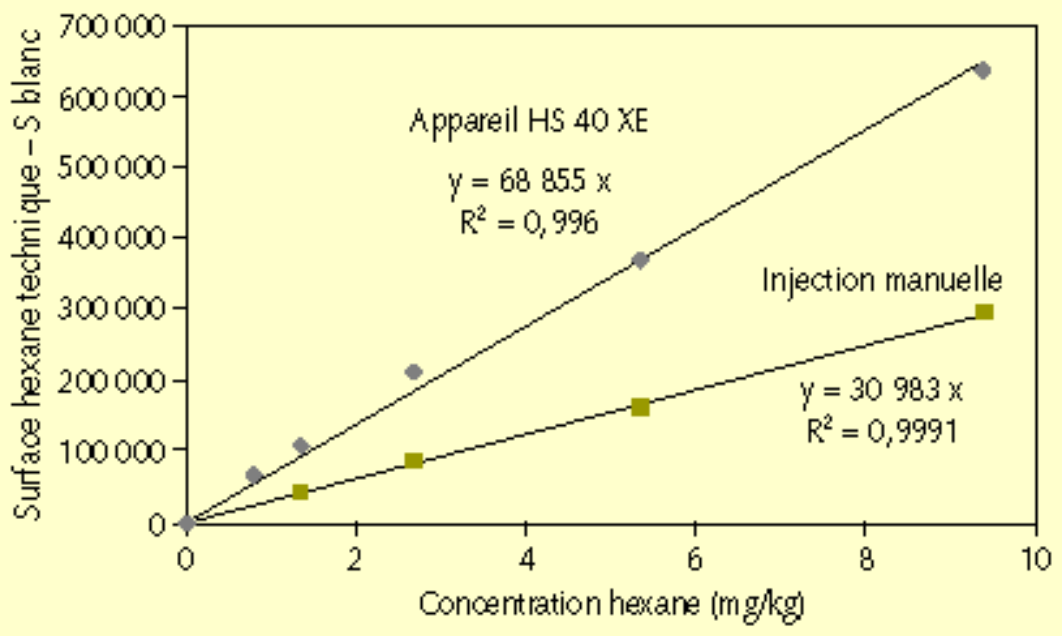

Figure 3. Appareil HS 40 XL - Droite d'étalonnage. 

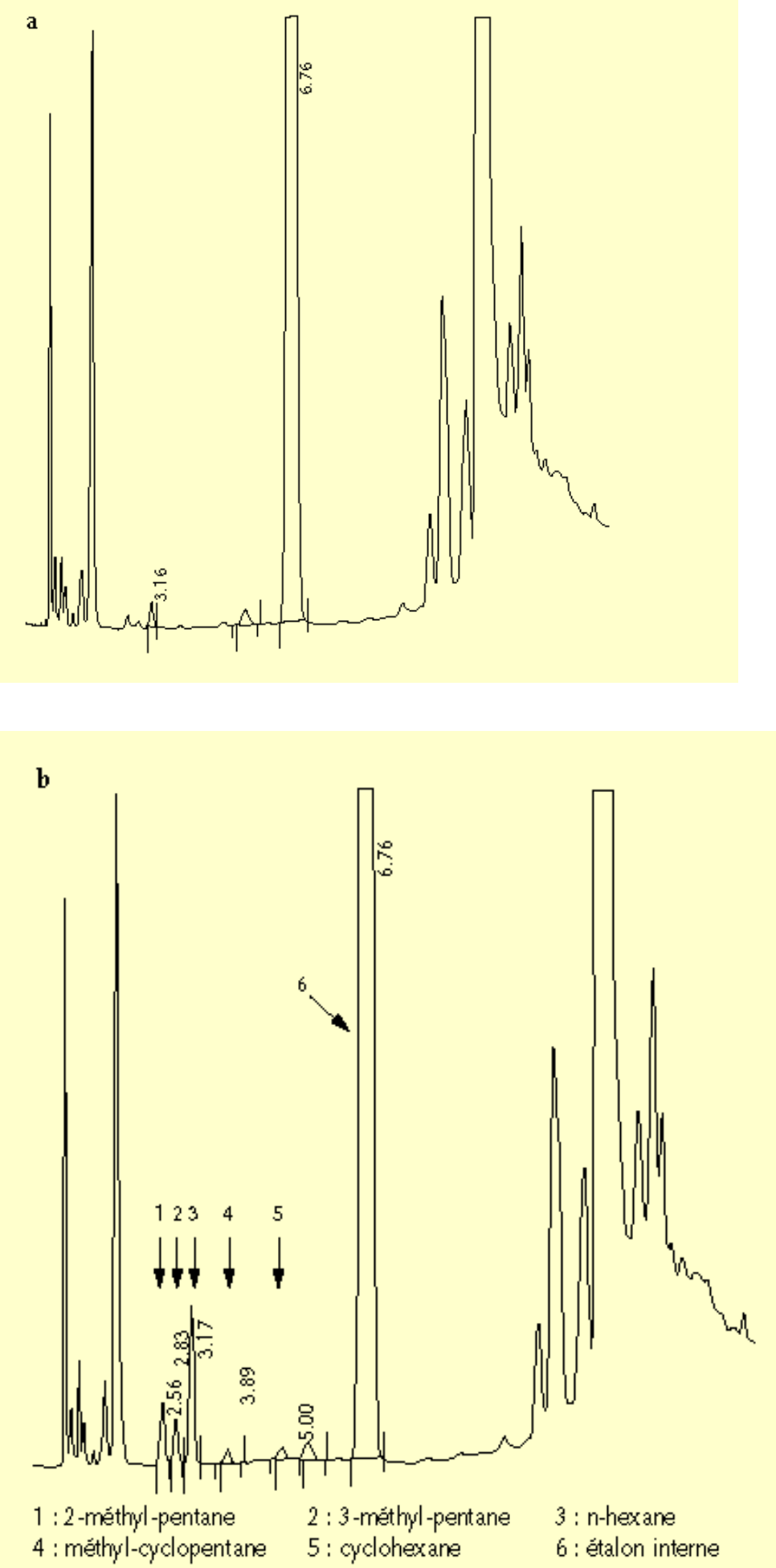

Figure 4. Profils chromatographiques obtenus avec le HS 40 XL. a. Huile de dilution. $b$. Échantillon complémenté à $0,10 \mathrm{mg} / \mathrm{kg}$. 


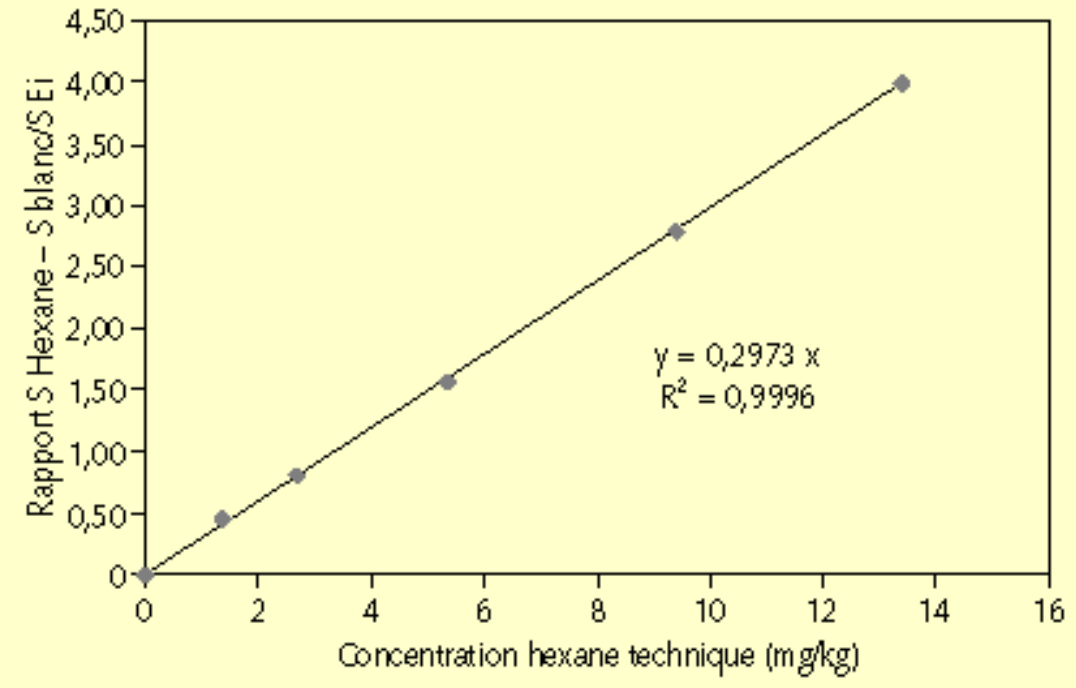

Figure 5. Injection manuelle sur colonne wide-bore. Linéarité de la réponse.

Tableau. Préparation des solutions de calitration.

\begin{tabular}{|c|c|c|c}
\hline $\begin{array}{c}\boldsymbol{\mu} \text { de solution } \\
\mathbf{d} \text { 'hex ane technique (8.2.1) }\end{array}$ & $\begin{array}{c}\text { Concentration en hexane technique } \\
(\mathbf{m g} / \mathbf{k g}): \mathbf{C h}\end{array}$ & $\begin{array}{c}\boldsymbol{\mu l} \text { de solution d'heptane } \\
\mathbf{( 8 . 2 . 2 )}\end{array}$ & $\begin{array}{c}\text { Concentration en heptane } \\
(\mathbf{m g} / \mathbf{k g}): \mathbf{C e i}\end{array}$ \\
\hline 0 & 0 & 5,0 & 6,84 \\
1,0 & 1,34 & 5,0 & 6,84 \\
2,0 & 2,68 & 5,0 & 6,84 \\
4,0 & 5,36 & 5,0 & 6,84 \\
7,0 & 9,38 & 5,0 & 6,84 \\
10,0 & 13,4 & 5,0 & 6,84
\end{tabular}


Tableau 1. Récapitulatif des conditions analytiques des méthodes testees.

\begin{tabular}{|c|c|c|c|c|}
\hline & Norme NF T 60-257 & $\begin{array}{l}\text { Appareil HS } 500 \\
\text { (CTC Analytios) }\end{array}$ & $\begin{array}{c}\text { Appareil HS } 40 \text { XL } \\
\text { (Perkin Emer) }\end{array}$ & $\begin{array}{l}\text { hjection manuelle } \\
\text { (colonne wide-bore) }\end{array}$ \\
\hline & \multicolumn{4}{|c|}{ Génération de l'espace de tête } \\
\hline $\begin{array}{l}\text { Vial } \\
\text { Prise d'essa i } \\
\text { Etabn interne } \\
\text { T' désorption } \\
\text { Durée désorption } \\
\text { Volume gazeux pré levé } \\
\text { Agitation } \\
\text { Temps de pressurisation } \\
\text { Temps d'injection } \\
\text { Temps de retrait } \\
T \text { ' de l'aiguille } \\
T \text { ' ligne de transfert }\end{array}$ & $\begin{array}{l}25 \mathrm{ml} \\
5 \mathrm{~g} \mathrm{~d} \text { huile } \\
5 \mathrm{mul} \text { heptane au } 1 / 100 \text { octane } \\
80 \text { 'C } \\
1 \mathrm{~h} \\
1 \mathrm{ml} \\
\text { Aucune }\end{array}$ & $\begin{array}{l}10 \mathrm{ml} \\
2 \mathrm{~g} \mathrm{~d} \text { huile } \\
5 \mathrm{mml} \text { heptane au } 1 / 250 \text { octane } \\
80 \mathrm{C} C \\
1 \mathrm{~h} \\
1 \mathrm{ml} \\
1000 \mathrm{rpm}\end{array}$ & $\begin{array}{l}22 \mathrm{ml} \\
5 \mathrm{~g} \mathrm{~d} \text { huile } \\
5 \mathrm{mul} \text { heptane au } 1 / 100 \text { octane } \\
80 \text { 'C } \\
30 \mathrm{~min} \\
2,4 \mathrm{ml} \\
\text { Aucune } \\
2 \mathrm{~min} \\
0,2 \mathrm{~min} \\
0,5 \mathrm{~min} \\
100 \text { 'C } \\
120^{\prime} \mathrm{C}\end{array}$ & $\begin{array}{l}25 \mathrm{ml} \\
5 \mathrm{~g} \mathrm{~d} \text { 'huile } \\
5 \mathrm{mul} \text { heptane au } 1 / 100 \text { octane } \\
80 \mathrm{C} \\
1 \mathrm{~h} \\
2,5 \mathrm{ml} \\
\text { Aucune }\end{array}$ \\
\hline T' ligne de transfert & \multicolumn{4}{|c|}{ Chromatographie en phase gazeuse } \\
\hline $\begin{array}{l}\text { Colonne } \\
T^{\prime} \text { 'injecteur } \\
T^{\prime} \text { 'détecteur } \\
T^{\prime} \text { 'four } \\
\text { Pression } \\
\text { Fuite } \\
\text { Débit }\end{array}$ & $\begin{array}{l}\text { DB1 30m, 0,32 mm, } 3 \text { mum } \\
150 \text { 'C } \\
150 \text { 'C } \\
50 \text { 'C (3 min) } \rightarrow 100^{~ ' C} \\
(1 \mathrm{~min}) \text { à } 10{ }^{\circ} \mathrm{C} / \mathrm{min} \\
0,7 \mathrm{bar} \text { (hydrogène) } \\
25 \mathrm{ml} / \text { min } \\
\text { Non mesuré }\end{array}$ & ldem norme NFT 60-257 & $\begin{array}{l}\text { DB1 30m, 0,53 mm, } 5 \mathrm{~mm} \\
120 \text { 'C } \\
150 \text { 'C } \\
50 \text { 'C (8 min) } \rightarrow 100 \text { 'C } \\
\text { (1 min) à } 10 \text { 'C/min } \\
0,6 \text { bar (hèlium) } \\
\text { Pasde fuite } \\
12 \text { mV/min }\end{array}$ & $\begin{array}{l}\text { DB1 30m, 0,53 mm, } 5 \mathrm{~mm} \\
150 \text { 'C } \\
200 \text { 'C } \\
50 \text { 'C (8 min) } \rightarrow 1200^{\circ} \mathrm{C} \\
(5 \mathrm{~min}) \text { à } 10 \text { ' } \mathrm{C} / \mathrm{min} \\
0,6 \mathrm{bar} \text { (hèlium) } \\
12 \mathrm{mV} \text { min } \\
13 \mathrm{mV} / \mathrm{min}\end{array}$ \\
\hline
\end{tabular}

El : étalon interne.

Tableau 2. Evaluation du facteur d'étalonnage en injection manuelle et avec le HS 500 (chromatographie survant nar me NF T 60-257).

\begin{tabular}{|l|c|c|}
\hline $\begin{array}{c}\text { Concentration hexane technique } \\
\text { (mg/kg) }\end{array}$ & F (HS 500) & F (manuelle) \\
\hline 1,3 & 0,60 & 0,54 \\
2,7 & 0,61 & 0,56 \\
5,4 & 0,64 & 0,55 \\
9,4 & - & 0,56 \\
F moyen & 0,62 & 0,55 \\
\hline
\end{tabular}

$$
F=W_{h} \times A_{d} /\left[\left(A_{t}-A_{t}-A_{\jmath}\right) \times W_{\downarrow}\right]
$$

A : surface totale (hexane + étabn interne)

A : surface étalon interne

A : surface hexane de l'huile de dilution

$W_{n}$ : tenes en hexane du point de gamme

$W_{\text {" }}$ : te neur en étalon interne du point de gamme

(hexane = somme des 5 isorries) 
Tableau 3. Evaluation du taux de récupération en injection manuele et avec le HS 500 (chromatog aphie suivant norme NF T 60-257).

\begin{tabular}{|l|c|c|c|c|}
\hline $\begin{array}{l}\text { Conc. hexane } \\
\text { technique théo rique (mg/kg) }\end{array}$ & \multicolumn{2}{|c|}{ Conc. hexane dosée (mg/kg) } \\
manuelle & \multicolumn{2}{c|}{ HS 500 } \\
\hline 0,25 & 0,46 & 0,28 & 184 & 112 \\
0,50 & 0,65 & 0,49 & 130 & 98 \\
0,76 & 0,85 & 0,64 & 112 & 84 \\
1,00 & - & 0,84 & - & 84 \\
\hline
\end{tabular}

Sastraction de a concentration de l'huile de dilution effectuée.

Tableau 4. Appareil HS $40 \mathrm{XL}$ - taux de récupération (moyenne de 5 injections).

\begin{tabular}{|c|c|c|c|}
\hline $\begin{array}{l}\text { Conc. hexame teo hnique théorique } \\
\qquad(\mathrm{mg} / \mathrm{kg})\end{array}$ & Conc. hexane tec hnique dosée & Taux de récupération (\%) & Coefficient de variation (\%) \\
\hline 0 & 0,06 & - & 12,4 \\
\hline 0,10 & 0,10 & 103 & 12,3 \\
\hline 0,25 & 0,22 & 89 & 5,0 \\
\hline 0,49 & 0,45 & 92 & 1,4 \\
\hline 0,74 & 0,65 & 88 & 4,8 \\
\hline 0,95 & 0,86 & 90 & 3,0 \\
\hline
\end{tabular}

Sast action de a concentr ation de l'hule de dilution effectuée.

Tablesu 5. Injection manuelle ss colonne wide-bore - taux de récupération. Domaine de concentration de 0,1 a $2 \mathrm{mg} / \mathrm{kg}$.

\begin{tabular}{|c|c|c}
\hline $\begin{array}{c}\text { Conc. hexane } \\
\text { technique théorique } \\
(\mathbf{m g} / \mathbf{k g})\end{array}$ & $\begin{array}{c}\text { Conc. hexane } \\
\text { technique } \\
\text { dosée }\end{array}$ & $\begin{array}{c}\text { Taux } \\
\text { de récupération } \\
\mathbf{( \% )}\end{array}$ \\
\hline 0 & 0,022 & - \\
0,099 & 0,111 & 112 \\
0,248 & 0,238 & 96 \\
0,530 & 0,533 & 100 \\
0,780 & 0,832 & 107 \\
1,034 & 0,984 & 95 \\
1,258 & 1,165 & 93 \\
2,022 & 1,989 & 98 \\
\hline
\end{tabular}

Sastraction de b concentration de l'bula de dilution effectueie.

Tableau 6. Injection manuelle sur colonne wide-bore - répétabilitié du dosage de l'hexane technique (moyenne de 5 injections).

\begin{tabular}{|l|c|c|c|}
\hline $\begin{array}{c}\text { Conc. hexane } \\
\text { technique } \\
\text { théo rique }(\mathbf{m g} / \mathbf{k g})\end{array}$ & $\begin{array}{c}\text { Conc. hexane } \\
\text { technique } \\
\text { dosée }\end{array}$ & $\begin{array}{c}\text { Taux } \\
\text { de récuperation } \\
\mathbf{( \% )}\end{array}$ & $\begin{array}{c}\text { Coeff icient } \\
\text { de variation } \\
\mathbf{( \% )}\end{array}$ \\
\hline Huile de dilution & 0,039 & - & - \\
0,248 & 0,268 & 108 & 0,7 \\
1,034 & 1,069 & 103 & 2,9 \\
\hline
\end{tabular}

Sastraction de a concentration de l'hule de dilution effectuée. 